\title{
21. LIGHT OF THE NIGHT SKY (LUMIERE DU CIEL NOCTURNE)
}

PRESIDENT : H. Tanabe VICE-PRESIDENT : R.H. Giese ORGANIZING COMMITTEE : R. Dumont, M. Harwit, C. Leinert, A.C. Levasseur-Regourd, K. Mattila, Yu.L. Trutse, J.L. Weinberg

\section{INTRODUCTION}

The light of the night sky includes several components which spread all over the celestial sphere. These light components are terrestrial (airglow), interplanetary (zodiacal light), galactic (integrated starlight, diffuse galactic light) and extragalactic (extragalactic background Iight). Thus the study of nature of each light source, covering large distance, is pursued in different fields of astronomy. However, the techniques of measurement for respective components are similar and the knowledge of other lights is indispensable even in the study of a particular component.

Historically, the early works on airglow were performed mostly by astronomers. In recent years, however, main part of airglow studies have been undertaken within the category of geophysics. Accordingly, we report here a limited number of airglow papers on photometric features of astronomical interest.

In August 27-30, 1979, IAU Symposium No.90 "Solid Particles in the Solar System" was held at Ottawa, Canada. Eleven invited papers and numerous contributed papers were presented on the zodiacal light, meteors and meteorites, interplanetary dust complex and planetary rings. All these papers are included in the Proceedings edited by Halliday and McIntosh (28.012.019) and those relevant to the field of our commission are summarized in this report.

In this triennium, as in previous one, considerable new knowledge has been gained in our field. Survey papers on the zodiacal light and interplanetary dust have been published by Stanley et al. (25.106.012), Millman (25.106.072), Brownlee (26.106.047), Giese (28.106.021) and Fechtig et al. (1981).

This report was prepared with help of Drs, Dumont, Giese and Mattila. Most of the references to USSR studies were taken from a report prepared by Dr. Shefov.

\section{AIRGIOW}

Spectrum and continuum. An airglow atlas covering the 0.9-2.3um spectral range was given by Steed and Baker (26.082.142) obtained from data taken on two separate nights - near local midnight of 3, 4 December 1975 - at White Sands Missile Range, New Mexico, using a field-widened interferometer-spectrometer.

Sobolev (1979) and Krassovsky et al. (1980) have shown that there is no increase of near IR continuum intensity (average: $I \mathrm{R} / \AA$ ) in comparison with visible region and intensity variation depends on the nitric oxide concentration in the mesopause. On the other hand, a high-latitude $\left(68^{\circ} \mathrm{N}\right)$ rocket measurement of $\mathrm{NO}_{2}$ continuum at $5400 \AA$ made by witt et al. $(29.082 .027)$ yielded an overhead intensity of $1.14 \mathrm{R} / \AA$.

During IR photographic airglow observations covering several years at Haleakala, Peterson (26.082.143) recorded three naked-eye events, which appear quickly, endure 0.5 - 1 hour, then fade. Visible photos of two events appear enhanced while little 
enhancement is present in the IR photos, although the structures are well correlated. He suggested that these events are due to either some unrecognized enhancement mechanism of the visible $\mathrm{OH}$ over the IR OH or an unrecognized continuum emitter.

5577 $\AA$ [OI]. From the global distribution of $5577 \AA$ on the night side of the earth observed by ISIS 2 satellite since 1971, Cogger and Khaneja (26.082.089) and Cogger and Murphree (28.082.068) identified the maximum occuring near $40^{\circ}$ latitude in winter and near $30^{\circ}$ in summer. Variation with time of year is small at low latitudes and increases towards middle latitudes where both a semiannual (maxima: April and October) and a seasonal (winter maximum) variations occur.

There are publications discussing about correlations with NaD and OH (Takahashi et al. (25.082.059), Clemesha et al. (26.082.117)), dependence on the solar activity (Fishkova (1981)), and drift of quasi periodic structure (Freund and Jacka (25.082. 014)). The developement of the theory was reviewed by Bates (1981).

$6300 \AA[O I]$. North-south aligned equatorial $6300 \AA$ airglow depletions were detected by Weber et al. (28.083.036) with an airborne all-sky imaging photometer. These depletions have east-west dimensions ranging $50-200 \mathrm{~km}$ with fine structure and often extend more than $1200 \mathrm{~km}$ north-south. The depletions appear after sunset and drift toward the east with speeds of $50-150 \mathrm{~m} / \mathrm{s}$ during the local evening hours. Existence of this phenomenon has been confirmed by Sahai et al. (29.082.078), Carman (1981) and Tanabe et al. (1981).

Meier (26.082.006) reviewed low latitude airglow phenomena observed with new techniques using high resolution interferometers and imaging devices during the past few years.

Lyman $\alpha$. An anomalous nighttime Lyman $\alpha$ emission in a latitude range $\pm 40^{\circ}$ was detected from satellites D2A ( $21 \mathrm{kR}$, Cazes and Emerich (28.082.064)) and OGO 6 (up to $8 \mathrm{kR}$, Thomas $(28.082 .065))$. The former authors suggested that the source of excitation is precipitation of particles, but the latter attributed to direct charged particle interference.

Night airglow of other planets. Atmospheric UV emissions from the night side of Venus observed by Pioneer Venus Orbiter (observed range: 1800-2800尺) and International Ultraviolet Explorer satellite (1850-2150 $)$ were identified as the bands of NO delta and gamma systems (Stewart and Barth (26.093.006), Feldman et al. (25. 093.055)). Stewart and Barth found that the airglow is brightest at equatorial latitudes, and at longitudes on the morning side of the antisolar meridian. Using a Fourier transform spectrometer and the $5 \mathrm{~m}$ Hale telescope, Connes et al. (26.093. 035) have detected an intense airglow from $\mathrm{O}_{2}\left({ }^{1} \Delta\right)$ at $1.27 \mu \mathrm{m}$, which is roughly 1000 times brighter than the visible $\mathrm{O}_{2}$ nightglow found by Veneras 9 and 10.

The Voyager UV spectrometer has made extensive observations of airglow from Jupiter's dark-side equatorial latitudes. The brightness of Lyman $\alpha$, the only emission detected, varies between 700 and 1000 as a function of longitude (McConnell. et al. $(28.099 .047))$.

\section{ZODIACAL LIGHT}

(a) Observations of the Zodiacal Light

Photometry, with or without polarimetry, in the visible and near-visible domain $(\sim 3000$ to $10000 \AA$ ) led to new results, obtained from space probes (Helios 1 and 2: Leinert et a.. (28.106.022, 1981a), and Leinert and Planck (1981)), from satellites (Explorer C: Torr et al. (26.106.017); Skylab: Weinberg and Hahn (28.106.023); Saliout 6: Lamy et al. (28.106.025)), from rocket (Astro 7: Pitz et al. (25.106.020)) 
and from high-altitude ground-based stations (Tenerife: Levasseur-Regourd and Dumont $(27.106 .014))$.

Although restricted to discrete off-ecliptic latitudes, Helios data (Leinert et al. (1981a, 1981b)) are among the most reliable and outstanding sources for our present view of the zodiacal cloud inside the earth's orbit. They have now been made available by Leinert et al. to the community in a convenient form via the NSSDC.

Skylab unfortunately gave less than expected data but in the few directions reported, the results are in good agreement with previous (space and ground-based) ones. Explorer $C$ results are the first, from any satellite, with such an extended celestial coverage; on some matters, they largely disagree with previous observations, e.g. the maximum found in the ecliptic near $130^{\circ}$ elongation, where a shallow minimum was generally found. The shortcomings of the (provisional) treatment, mainly with respect to starlight, which is optimistically considered to be less worrying than from ground, have to be kept in mind as whether the reported features are real.

The table of zodiacal brightnesses versus helioecliptic coordinates, averaged from Tenerife data, is the first to be in fair agreement with the bulk of spaceborne brightness data, and to be smoothed sufficiently well so as to give access to the angular derivatives whose knowledge is required in the new inversion methods. The implications of this celestial distribution of zodiacal brightness upon the expected $\mathrm{s} / \mathrm{n}$ ratio in the space detection of very faint objects - e.g. with the Space Telescope - have been studied by Dumont and Levasseur-Regourd (1981).

Several publications, e.g. Asaad et al. (1979a), witness a strong increase of zodiacal observational activity at Helwan Observatory, Egypt.

Photometry in the UV domain showed an increase of agreement in a formerly controversial matter. From observations during the Apollo 17 mission, Henry et al. (28.106.026) unsuccessfully tried to detect zodiacal light in the far-UV (1180 to $1680 \AA)$, and derived for that component upper limits considerably below the high level formerly supported by $\mathrm{OAO} 2$ observers. Rather similar conclusions arose from the work of Maucherat-Joubert et al. (25.106.022) at $1690 \AA$ with the satellite D2B, and from the work of Morgan (22.106.048) at $1550 \AA$ with the satellite TD-1. From 1800 to $2600 \AA$, Pitz et al. (25.106.020) found the zodiacal light redder than the sun; they also found the polarization degree higher in the UV than in the visible.

Photometry in the IR (thermal) domain is a subject where previous results were very scarce. Of major interest is the contribution by Price et al. (27.106.028) giving the thermal emission of interplanetary dust near 11 and $20 \mu \mathrm{m}$ in a whole zodiacal cone: $\lambda-\lambda_{\odot}=35$ to $75^{\circ} ;|\beta|<30^{\circ}$. A conspicuous similarity appears between these IR gradients versus elongation, and those observed in the visual domain.

Very few works have been reported in zodiacal light spectrometry, except the fact that a spectrophotometric study, with purpose of detecting Doppler shifts, is in progress at Pic-du-Midi Observatory.

(b) International Solar Polar Mission: Zodiacal Light/Background Starlight Experiment (ISPM/ZLE)

The planned ISPM/ZLE space experiment (Schwehm (28.051.012), Schwehm et al. (1981)) involving many Commission 21 members was a photopolarimeter of $4 \mathrm{~cm}$ in aperture based on a coronagraph optics like to zodiacal light experiment on Helios. It was planned to measure, for elongations between $30^{\circ}$ and $180^{\circ}$, intensity and linear polarization in 6 wavelength bands between $1800 \AA$ and $3500 \AA$ and in 6 bands between $3500 \AA$ and $8000 \AA$ during the trip of ISPM out to Jupiter, above the ecliptic plane (up to more than $2 \mathrm{AU}$ ), and while the spacecraft would return to and cross the ecliptic 
plane near 1.2 AU in solar distance. This experiment was an unique opportunity to probe the zodiacal cloud in three dimensions (Giese (26.106.014)).

It was funded mainly by the German Ministery of Research and Technology (BMFT) and prepared by the Bochum group in cooperation with the Albany/Gainesville group and JPL supported by NASA. After selection of Dornier as contractor, work on ZLE proceeded fast: the lab-unit was completed, the preliminary design review took successfully place at Friedrichshafen 1980 and most of the engineering unit was completed in autumn 1981. Launch was scheduled for early 1986.

Unfortunately, however, NASA cancelled the US-spacecraft, for which ZLE was booked, for budget reasons in October 1981: there will be no photopolarimeter on the ISPM.

\section{(c) Interpretations of Zodiacal Light Observations}

(i) Macroscopic features of the zodiacal cloud

The heliocentric dependence of the dust space density in the symmetry plane of the zodiacal cloud has mainly been studied through Helios 1 and 2 data (Leinert et al. (28.106.022, 1981a), Leinert and Planck (1981)). The value $2.3 \pm 0.05$ of the exponent for the variation of zodiacal brightness versus probe's heliocentric distance, implies the exponent 1.3 for the variation of density versus heliocentric distance, both in or near the ecliptic. That the same value is obtained in all of the observed directions is a strong argument in favour of a smooth distribution and a homogeneous composition of the dust. Homogeneity is also corroborated by colour, which for a fixed viewing direction remains independent of solar distance. On the other hand, however, a decrease of polarization with decreasing solar distance suggests some changes of grain properties not yet satisfactorily explained (Leinert et al. (28.106.022, 1981a)). There is also some evidence, that beyond 1 AU the properties of dust might not be homogeneous (Schuerman (28.106.029)).

The off-ecliptic distribution of dust has been discussed by Helios investigators (Leinert et al. (1981a)), who favour an exp $(-2.1|z / r|)$ elevation dependence.

Interesting suggestions have been made in order to disentangle from the observations the $f\left(B_{\odot}\right)$ function (assumed to represent the latitudinal decrease of density at a given heliocentric distance) by Buitrago et al. (29.031.505) for earthbound measurements, and by Dumont and Levasseur-Regourd (28.031.543) in the case of ISPM.

Brightness and/or polarization changes, possibly due to local inhomogeneities, have been searched in Helios data with the conclusion that these features, if any, are below the level of detectability. It was not possible to find evidence for local concentrations of dust related to meteor streams (Leinert et al. (1981a)); for solar-cycled variations of zodiacal brightness (Leinert et al. (28.106.022)); nor for the instabilities and asymmetries of the polarization in the antisolar cap (Leinert and Planck (1981)), previously advocated by some ground-based observers.

Robley $(26.106 .020,28.106 .024)$ reported a sudden change, near 1968, in the brightness of the Gegenschein, as observed from Pic-du-Midi, and related this event to solar wind variation. Asaad (1979) examined some possible explanations for the discrepancies between reported zodiacal light brightnesses.

Contributions to the problem of Libration clouds can be found in Mercer et al. $(25.106 .045)$ and in Kordylewski $(28.106 .074)$.

The plane(s) of symmetry of the zodiacal cloud were throughly investigated. For the inner part of the solar system (0.1 to I AU), Leinert et al. (27.106.003, 1981a) derived from Helios data that the plane of symmetry is represented throughout 
this region by one single plane $i=3.0^{\circ}$ and $\Omega=87^{\circ}$ with respect to the ecliptic. For the region beyond I AU, Tanabe et al. (28.106.027) derived a plane $i=0.47^{\circ}$, $\Omega=78.9^{\circ}$ as the best-fit symmetry plane for observed position of the Gegenschein reported by various observers. They showed that this symmetry plane is consistent with their photometric axis measurement of the zodiacal light at large elongations $\left(90^{\circ}<\lambda-\lambda \odot<270^{\circ}\right)$.

Misconi (28.106.028), based on Hawaii data, suggested that there exists a surface of symmetry (rather than one plane of symmetry), which changes from being close to the orbital plane of Venus inside to close to the invariable plane near $1 \mathrm{AU}$. Recently, Misconi (1981) showed from his model calculations that the backscattering effect $\left(\theta \sim 165-180^{\circ}\right)$ overrides the space distribution effect of the dust, and the photometric center of the Gegenschein should be observed at the antisolar point at all times, except possibly when the antisolar point is at its maximum displacement from the symmetry plane.

(ii) Physical properties of grains: models and inversion techniques

Optical properties of zodiacal dust are mainly inferred from the volume scattering intensity and polarization curve as a function of scattering angle, both inverted from brightness versus elongation data under the assumption of a $r^{-1} .3$ heliocentric density law: thus observational and laboratory results can be directly compared.

From a "Comet fragment model for zodiacal particles" Greenberg and Gustafson (29.106.001) arrived at agglomerates ('birds nests') of small elongated and dielectric building stones, which show similar scattering properties as interplanetary dust (see also Gustafson (28.063.052)). The other approach (see Giese (28.106.021)) by absorbing fluffy particles (Brownlee particles), which also approximates interplanetary dust scattering, was extended to microwave simulation of agglomerates inhomogeneous in material by the Bochum group (Zerull et al. (1980)) in addition to laser scattering measurements of large (40 to $120 \mu \mathrm{m}$ ) irregular and meteoritic grains, which even seem to approximate interplanetary dust scattering including the forward directions (Weiss (1981)).

General scattering investigations have been continued by the Albany/Gainesville group (Schuerman et al. (1981), Schaefer (1980)) and among others by Mukai et a.l. (1980), Chiappetta $(27.063 .014)$. Staude and Röser (28.106.031) gave further developement to their models based on Mie theory.

Based on dust fluxes (of Morrison and Zinner) Lamy and Perrin (28.106.030) proposed a bimodal population model, where submicron particles have a non-neglible role. There remains some controversy about the fluxes of submicron particles and about the albedo of interplanetary grains. Since surprisingly low ( $\mathrm{A}=0.006$, Cook (21.106. 003)) and rather high $(A=0.24$, Hanner $(28.106 .081)$ ) albedos were reported in the literature, Hanner et al. (1982) started to rediscuss the problem taking into account enhanced scattering of fluffy particles as observed in the laboratory.

Dynamics and physical interactions of grains influence size and space distribution of the zodiacal grains. Investigations relevant to Commission 21 were performed in the field of interaction with solar radiation (Schwehm (1979, 1980), Burns et al. (26.106.016)) and particles (Mukai and Schwehm (29.106.006)), modification of the three body problem by radiation effect (Schuerman (27.042.072)) and interaction of interstellar grains with the interplanetary environment (Gustafson and Misconi (26.131.100), Morfill and Grin (28.131.116)).

Inversion of the brightness integral plays an increasing role in clarifying zodiacal light photopolarimetric results. Obtaining the brightness contribution and the local polarization degree of the final section of the line of sight is completely solved now. By very different ways, Dumont et al. (25.031.601) and Schuerman 
(25.106.017) have shown the existence of an "inversion plane" in which this inversion only requires "slightly arbitrary" (if any) assumptions. Other equivalent mathematical treatment was suggested by Buitrago (25.106.055). These methods were used by Schuerman (25.106.043, 28.106.029) to invert Pioneer 10 and 11 observations.

The extension of the inversion to the remaining of the line of sight is known to require theoretically physical assumptions, which Dumont and Levasseur-Regourd (28.031.543) tried to maintain at the lowest "uncertainty level" in the case of ISPM/ZLE data. Mujica et al. (28.106.002) also tried to limit the concessions to physical assumptions, replacing them as much as possible by purely mathematical hypotheses. In a similar fashion, Dumont and Pelletanne (1981) have shown the existence of two peculiar points on each line of sight, in the ecliptic, where the local optical properties can be derived with a very low level of physical assumptions, and with a very low sensitivity to mathematical ones.

\section{INTEGRATED STARLIGHT AND DIF'FUSE GALACTIC LIGHT}

Integrated starlight (ISL), including in some cases the diffuse galactic light (DGL). Progress on the Pioneer 10 and $l 1$ background starlight ( $B$ and $R$ bands) program has been reported by Weinberg (29.113.006), and Toller (28.155.039, 1981). Using the Helios 1 and 2 space probes Leinert and Richter (198.1) have measured the UBV intensities and colours along eight strips across the Milky Way. The calibration appears to be very reliable and the Helios results have therefore been used to test previous all sky photometric maps of the Milky Way. Tanabe and his associates have continued star counting on the POSS; ISL data is now available for a total area of $48.02 \mathbf{0}^{\circ}$. Zavarzin (1981) has observed photoelectrically the distribution of $V-R$ colour over $5^{\circ} \leqq 1 \leqq 240^{\circ},-20^{\circ}<\mathrm{b}<20^{\circ}$. Asaad et al. (1979b) have made photoelectric BVR measurements over a large latitude range and compared their data with Pioneer 10 and Tokyo starcount data. A photographic photometry of the Northern Milky Way in $U$ has been carried out by Winkler et al. (29.155.044).

In the far-UV region the ISL intensity has been computed by Gondhalekar et al. (27.157.003) in four pass bands at $1565 \AA, 1965 \AA, 2365 \AA$ and $2740 \AA$ on the basis of the ESRO TD-1/S2/68 satellite UV catalog containing 58012 stars. Direct measurements of the galactic UV radiation (ISL + UGL) have been reported by Anderson et al. ( 26 . 142.144), Pitz et al. (25.157.007), Henry et al. (28.157.006) and Paresce et al. (25. $142.064)$ in the 1200-2611 $\mathrm{A}$ region and Sandel et al. (25.131.074) in the 912-1200A region. Serious disagreements exist among observers regarding the intensity and the spatial variability of the radiation.

Surveys of the large scale IR emission near the galactic equator were reviewed by Okuda (29.156.006) for the observational side and by Drapatz (29.155.026) regarding their interpretation in terms of galactic star and dust distribution models.

Photometric Milky Way models by Caplan and Grec (26.155.013) and Mattila (27. 031.512, 27.155.009) predict the intensity and colour-index of the ISL. They can be useful in combination with observational ISL data.

The diffuse galactic light (DGL) includes (1) the scattered light from interstellar dust and possibly two new sources which are important in UV, namely: (2) emission due to interstellar $\mathrm{H}_{2}$ fluorescence (see Duley and Williams (28.131.336)) and (3) emission by hot galactic coronal gas (see Jakobsen and Paresce (29.157.007), Feldman et al. (1981)).

Henry (29.157.005) has observed the DGL at $1180-1680 \AA$ near $b=0^{\circ}$ using the Apollo 17 spectrometer. He found a high albedo, A $>0.5$, and a strongly forward scattering phase function, $g>0.7$. An important new observational result is the correlation between observed UV background intensity and the line of sight HI column 
density (see Paresce et al. (28.142.053)), Maucherat-Joubert et al. (28.157.002)). This indicates that a major part of the UV background radiation has a galactic origin even at high galactic latitudes. Jakobsen (1981) inerpreted the data in terms of scattering by high-lying dust clouds plus fluorescence emission of interstellar $\mathrm{H}_{2}$.

\section{EXTRAGALACTIC BACKGROUND LIGHT}

The extragalactic background light (EBL) and its cosmological implications have been reviewed by Leinert (25.162.008) in the optical, Paresce and Jakobsen (28.142.070) in the UV and Harwit (25.133.005) in the IR part of spectrum. Besides the integrated light of distant galaxies and quasars, also intergalactic stars and emission by hot plasma may contribute to the EBL (see Stabell and Wesson (28.162. 098), Jakobsen (27.142.018), Bussoletti et al. (27.051.049)).

The far-UV background at high galactic latitudes may contain a considerable portion of $E B L$. After subtraction of the galactic and other foreground components, there remains an intensity of $150-300$ photons $\left(\mathrm{cm}^{2} \mathrm{~s} \text { sterad } \AA\right)^{-1}$ at $1200-1600 \AA$, which may be ascribed to EBL (see Feldman et al. (1981), Paresce et al. (28.142.053), Maucherat-Joubert et al. (28.157.002), Anderson et al. (26.142.144)). But because of the uncertainties in foreground subtraction this must be considered only as an upper limit at present.

In the optical part of spectrum Dube et al. (26.162.005) have published an extensive discussion of their EBL measuring technique, and they derive an upper limit of $3.4 \mathrm{~S}_{10}$ at $5115 \AA$ (90\% confidence level). Spinrad and Stone (22.162.098) have repeated the EBL measurement near LI34 using Mattila's (17.131.034) dark cloud technique and they derived a $3 \sigma$ upper limit of $5 \mathrm{~S}_{10}$ at $4000 \AA$, in contradiction with Mattila's value of $I(E B L)=10 \pm 4 \mathrm{~S}_{10}$. Schnur and Mattila also repeated the measurement near L134 (see Schnur (1980)) and their observed sky brightness in and near I134 contradicted Spinrad and Stone's values but the data was insufficient to derive an EBL intensity value.

In the IR part of spectrum Fabbri and Melchiorri (26.031.523) have proposed a new technique for separating the EBL from the foreground components. It utilizes the screening effect of an X-ray cluster of galaxies on the IR background.

\section{CONCLUSIONS}

The cancellation of the ISPM/ZLE project is a matter of great regret. Considering a number of excellent results obtained by Helios 1, 2 and Pioneer 10,11 space probes, which flew only in or near the ecliptic plane, we were expecting that the out-of-ecliptic observation by the ISPM/ZLE would provide new discoveries and knowledges to the field of Commission 21. Nevertheless it is a good harvest that the ISPA/ZJE project has induced and activated many other internationally cooperative works in both experimental and theoretical studies. We hope that the networks of such various kinds of cooperation will expand and become more active, and further that the next opportunity of the out-of-ecliptic space observation will occur in the near future.

It is evident that the tropical region is the most suitable site for the groundbased zodiacal light observation. However, new techniques of observation have revealed low latitudes airglow phenomena previously unobserved. Accordingly, the subtraction of the airglow component must be made with care the zodiacal light observation, particulary for its faint (large elongation) part. More study of the airglow continuum; spectral distribution and its variation, is required.

Among the numerous results, developement of the inversion technique of the 
zodiacal brightness and the study of light scattering by irregularly shaped dust particles have made a substantial progress in this triennium. They provide useful contribution to detailed interpretation of the zodiacal light observation.

Photometric results of the galactic and extragalactic components are yet insufficient in comparison with those of other components due to faintness of the light, despite of various efforts for more accurate measurements. It is highly important to develope new high-speed techniques for star counts of wide sky area, and new ideas (some are mentioned in this report) for observation.

\section{REFERENCES}

Only papers with no Reference No. of Astron. Astrophys. Abstracts are listed.

Asaad, A.S. : 1979, J. Astron. Soc. Egypt, 1, p. 84.

Asaad, A.S., Mikhail, J.S., Nawar, S. : 1979a, Helwan Obs. Bull., No. 197.

Asaad, A.S., Mikhail, J.S., Abd El-Shaheed, S.N. : 1979b, Helwan Obs. Bull., No. 200.

Bates, D.R. : 1981, Planet. Space Sci., 29, p. 1061.

Carman, E.H. : 1981, IAGA Bull., No. 45, p. 350.

Dumont, R., Levasseur-Regourd, A.C. : 1981, Adv. Space Res., I, p. 127.

Dumont, R., Pelletanne, B. : 1981, Compt. Rend. Acad. Sci. Paris, in press.

Fechtig, H., Leinert, C., Grün, E. : 1981, in "Landolt-Börnstein, New Series, Group VI, Vol. 2a" (eds. K. Schaifers and H.H. Voigt), p. 228, Springer BerlinHeidelberg-New York.

Feldman, P.D., Brune, W.H., Henry, R.C. : 1981, Astrophys. J. Letters, in press.

Fishkova, L.M. : 1981, Aurorae and Airglow, N 29, p. 17, Acad. Sci. USSR.

Hanner, M.S., Giese, R.H., Weiss, K., Zerull, R.H. : 1982, Astron. Astrophys., in press.

Jakobsen, P. : 1981, preprint.

Krassovsky, V.I., Rapoport, Z. Ts., Smenov, A.I., Sobolev, V.G., Shefov, N.N. : 1980, Geomagnetism and Aeronomy, USSR, 20, N 4, p. 657.

Leinert, C., Richter, I., Pitz, E., Planck, B. : 198la, Astron. Astrophys., in press.

Leinert, C., Pitz, E., Link, H., Salm, N. : 1981b, J. Space Instrumentation, 5, p. 257.

Leinert, C., Planck, B. : 1981, Astron. Astrophys., in press.

Leinert, C., Richter, I. : 1981, Astron. Astrophys., in press.

Misconi, N.Y. : 1981, Icarus, in press.

Mukai, S., Mukai, T., Giese, R.H. : 1980. in "Light Scattering by Irregularly Shaped Particles" (ed. D.W. Schuerman), p. 219, Plenum Press, New York.

Schaefer, R. : 1980, Ph.D. Thesis, State Univ. New York Albany.

Schnur, G. : 1980, ESO Workshop on Two Dimensional Photometry (eds. P. Crane and K. Kjär), p. 365 .

Schuerman, D.W., Wang, R.T., Gustafson, B.A.S., Schaefer, R. : 1981, Appl. Opt., in press.

Schwehm, G. : 1979, Ph.D. Thesis, Ruhr-Univ. Bochum / 1980, BMFT-FB-W 79-40.

Schwehm, G., Giese, R.H., Giovane, F., Schuerman, D.W., Weinberg, J.L. : 1981, Adv. Space Res., 1, p. 121.

Sobolev, V.G. : 1979, Astron. Circular, USSR, N 1083, p. 7.

Tanabe, H., Takechi, A., Miyashita, A. : 1981, IAGA Bull., No. 45, p. 352.

Toller, G. : 1981, Ph.D. Thesis, State Univ. New York Stony Brook.

Weiss, K. : 1981, Ph.D. Thesis, Ruhr-Univ. Bochum.

Zavarzin, Ju. M. : 1981, Trudy Astrophys. Inst. (Alma-Ata), Vol. 38.

Zerull, R.H., Giese, R.H., Schwill, S., Weiss, K. : 1980, in "Light Scattering by Irregularly Shaped Particles" (ed. D.W. Schuerman), p. 273, Plenum Press, New York, London.

H. Tanabe

President of the Commission 